\title{
A Novel, Safe Way of Removing Non-Deflatable Bladder Catheter
}

\author{
Ziauddin Khan ${ }^{1}$, Umair Tahir Khan ${ }^{2}$, Imran Khan ${ }^{3}$, Tahir Naeem Khan ${ }^{4}$ \\ ${ }^{1}$ Consultant in Urology, Imam Abdulrahman Bin Faisal Hospital, Dammam, KSA \\ ${ }^{2}$ University of Leicester, Leicester, UK \\ ${ }^{3}$ Department of Radiology, Kings College Hospital London, London, UK \\ ${ }^{4}$ Department of Pathology, Northampton General Hospital, Northampton, UK \\ Email: ziauddink@hotmail.com
}

Received August 4, 2012; revised September 3, 2012; accepted September 13, 2012

\begin{abstract}
Non-deflated balloon of a transurethral inserted bladder catheter is a problem that could be difficult to manage, especially in males. A variety of methods have been described to puncture the balloon and remove the catheter, though these can be associated with complications. We illustrate a simple and a safe method to overcome this problem using a flexible cystoscope under local anaesthesia.
\end{abstract}

Keywords: Balloon Dilation; Urinary Catheterization; Urinary Bladder; Catheters; Equipment Failure

\section{Introduction}

Failure to deflate the balloon of a urinary catheter is a well recognized and often frustrating complication. Deflation of the balloon is relatively easier in females compared to males due to the short urethra. Failure to deflate the balloon could be due to faulty inflation valve, obstruction of balloon inflating channel intraluminally by crystal formation or a primary problem with the balloon $[1,2]$. Many methods have been described ranging from over distension of the balloon with water to the instillation of chemical through the balloon channel to more invasive methods using suprapubic, rectal or perineal routes to puncture the catheter balloon [3,4]. Methods using balloon drainage without external pressure with the help of guide-wire or ureteric guide-wire have been used also [5,6]. Different endoscopic methods have also been described $[7,8]$. If all these methods are unsuccessful then radiological puncture under ultrasound guidance is the method of choice [9]. All these methods have their own complications and difficulties and there is a need of simple bed-side procedure to resolve it. We describe our initial experience of dealing with non-deflated balloons using a flexible cystoscope under local anaesthesia.

\section{Technique}

In case of non-deflated balloon, the in situ catheter is gently pulled. Then the proximal end of the catheter lying outside the urethra is stitched with two separate stay sutures with No. 1 prolene at 2 points $15 \mathrm{~mm}$ apart (Fig- ure 1(a)) distal to the urethral meatus. The needles are cut and a clip is attached to the ends of each suture. Full lengths of the sutures are left. Subsequently, the catheter is cut distal to the sutures (Figure 1(a)). The proximal cut part of the catheter is then removed leaving the distal cut catheter lying in urethra and bladder with sutures at the end of it.

The urethra is lubricated and anaesthetised using $22 \mathrm{ml}$ of Instillagel (Farcopharma GMBH 50829 Köln) before introducing a flexible cystoscope (size 17 Charrièr (Ch.), external diameter). The cystoscope is introduced gently, to push the cut catheter into the bladder lumen while prolene is hanging outside the urethral meatus (Figure 1(b)). Once the balloon is visualized, an eXcelon ${ }^{\mathrm{TM}}$ (transbronchial aspiration needle M005 6412, Boston Scientific USA, size $6 \mathrm{Ch}$.) is introduced through the biopsy channel to puncture the balloon. The deflation is confirmed via direct visualization after which the scope is removed. Finally, the catheter remnant is retrieved by gently pulling on the Prolene sutures. The process is summarised in Figures 1(a)-(c).

\section{Discussion}

Although a non-deflating balloon is a rare problem, it poses a technical difficulty and is frustrating for the clinician [10] and the patient. Several techniques have been described in the literature to deal with this dilemma, though these are not without inherent problems. Ethyl chloride is no longer routinely available and chemical 


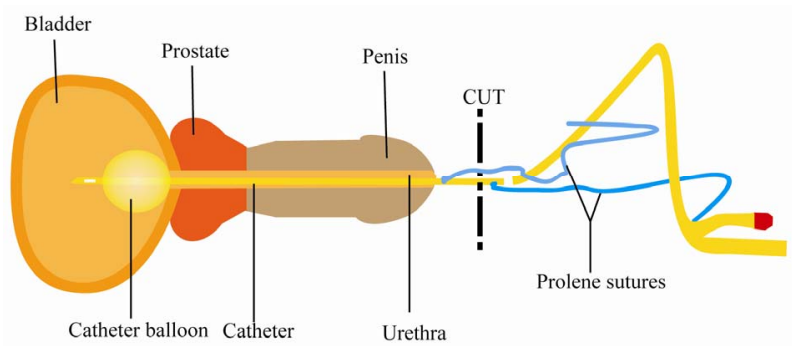

(a)

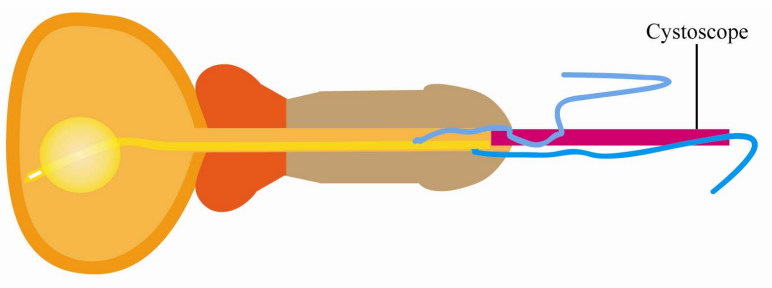

(b)

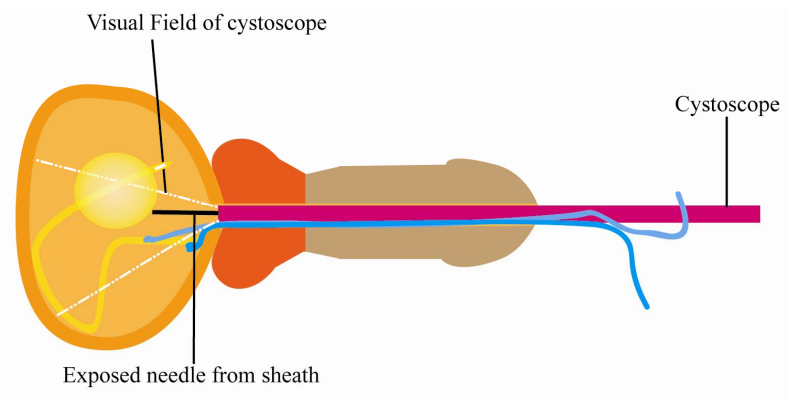

(c)

Figure 1. (a) The catheter is cut at the proximal end after sutures are stitched onto the catheter distal to the urethral meatus; (b) The cystoscope is inserted slowly which pushes the catheter into the bladder whilst the sutures hang out of the urethral meatus; (c) The cystoscope is advanced towards the bladder lumen whilst the sutures may be pulled slightly to ensure the catheter balloon is visualized. The needle is then exposed from sheath before puncturing the balloon. The sutures are then pulled gently to retrieve the catheter.

rupture of the balloon with it may cause irritation of the bladder mucosa [11] leading to chemical cystitis [12]. In addition, balloon fragments can be retained in the bladder leading to unnecessary cystoscopy. Suprapubic puncture of the balloon is often performed blindly in clinical settings and is invasive. It can be associated with complications including bowel injury and can be technically difficult in overweight patients. Use of a cannula and a syringe has also been described, however this is also a blind process [13]. The passage of a stylet via the balloon inflation channel as described by Sood and Sahota [14] can be traumatic to the urethra.

It is therefore essential to have an effective yet simple bed-side method for dealing with this problem. We feel that our technique provides a simple and reliable method.
It is minimally invasive, safe and reproducible and can be performed at the bedside. So far, we have performed this procedure on 6 patients without any failures or complications and more importantly achieved patients' satisfaction with the technique. The added expense of the disposable needle is worth the benefit it affords without any complications. The prolene sutures can theoretically cut through the catheter when pulling but two sutures with a distance of $15 \mathrm{~mm}$ between them on either side affords necessary protection against failure.

\section{REFERENCES}

[1] P. C. C. Pitt, "Self Retained Catheters," British Journal of Hospital Medicine, Vol. 4, 1970, p. 174.

[2] T.-C. Lin, H. L. Shieh, M. S. Lin, C. T. Chen, C. S. Wu and Y. C. Lin, "An Alternative Technique for Deflation of a Non-Deflating Balloon in a Small Calibre F8 Foley Catheter in Women," JTUA, Vol. 20, No. 1, 2009, pp. 3233.

[3] R. Moskovich, "Suprapubic Puncture for Non-Deflating Urethral Balloon. A Safe Method of Deflation," Journal of the Royal College of Surgeons of Edinburgh, Vol. 29, No. 3, 1984, pp. 181-183.

[4] L. E. Moffat, C. Teo and I. Dawson, "Ultrasound in Management of Undeflatable Foley Catheter Balloon," Urology, Vol. 26, No. 1, 1985, p. 79. doi:10.1016/0090-4295(85)90265-1

[5] R. K. Godwin and S. M. Lloyd, "The Non-Deflating Foley Catheter," British Journal of Clinical Practice, Vol. 44, No. 11, 1990, pp. 438-440.

[6] H. T. Bui, D. Agarwal and A. Clarke, "An Easy Method of Deflating a Blocked Foley Balloon," Australian New Zeal K Surgery, Vol. 72, No. 11, 2002, p. 843. doi:10.1046/j.1445-2197.2002.02551.x

[7] K. J. O'Flynn, D. G. Thomas and A. Hardy, "Harpoon Device for the Removal of Obstructed Balloon Catheters," British Journal of Urology, Vol. 69, No. 2, 1992, p. 217. doi:10.1111/j.1464-410X.1992.tb15511.x

[8] A. Saxena, S. Khanna and B. K. Vohra, "Endoscopic Management of the Undeflateable Foley's Catheter Balloon," British Journal of Urology, Vol. 69, No. 2, 1992, pp. 217-218. doi:10.1111/j.1464-410X.1992.tb15512.x

[9] R. Patterson, B. Little, J. Tolan and C. Sweeney, "How to Manage a Urinary Catheter Balloon That Will Not Deflate," International Journal of Urology \& Nephrology, Vol. 38, No. 1, 2006, pp. 57-61. doi:10.1007/s11255-005-2945-7

[10] S. St Luce, A. C. Ninan, J. A. Hall, I. J. Kimberl, J. A. Petros and M. M. Issa, "Role of Transrectal Ultrasonography in Diagnosis and Treatment of Retained Foley Catheter," Urology, Vol. 65, No. 5, 2005, p. 1001. doi:10.1016/j.urology.2004.11.010

[11] H. U. Eickinberg, M. Amin and J. Clark, "Non-Deflating Foley's Catheter," International Journal of Urology \& Nephrology, Vol. 8, No. 2, 1976, pp. 171-173. doi:10.1007/BF02082214 
[12] S. Khanna, "Preliminary Results for Toluene for Retained Urinary Catheters," European Urology, Vol. 19, No. 2, 1991, pp. 169-170.

[13] T. S. Huseyin and S. M. Moalypour, "A Solution to the Non-Deflating Balloon of a Suprapubic Catheter," Emergency Medicine Journal, Vol. 19, No. 4, 2002, p. 354.

\section{Author Contribution}

Ziauddin Khan: Carried out the procedures, literature search and wrote the manuscript.

Umair Tahir Khan: Illustrated and designed the figures, carried out literature search and co-wrote the manuscript. Imran Khan: Co-wrote the manuscript.

Tahir Naeem Khan: Co-wrote the manuscript and carried out review of literature.

\section{doi:10.1136/emj.19.4.354}

[14] S. C. Sood and H. Sahota, "Removing Obstructed Balloon Catheter," British Medical Journal, Vol. 4, No. 5842, 1972, p. 735. doi:10.1136/bmj.4.5842.735-d 